Article

\title{
Intense Breeding within Lentil Landraces for High-Yielding Pure Lines Sustained the Seed Quality Characteristics
}

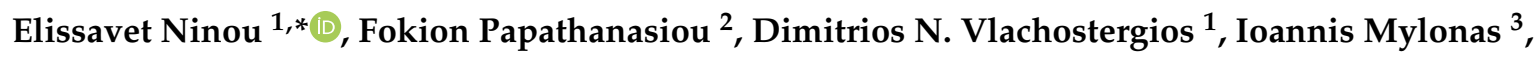 \\ Anastasia Kargiotidou ${ }^{1}$, Chrysanthi Pankou ${ }^{4}$, Ioannis Papadopoulos ${ }^{2}$, Evangelia Sinapidou ${ }^{5}$ \\ and Ioannis Tokatlidis 5 \\ 1 Institute of Industrial and Forage Crops, Hellenic Agricultural Organization Demeter, 41335 Larissa, Greece \\ Department of Agriculture, University of Western Macedonia, 53100 Florina, Greece \\ 3 Institute of Plant Breeding and Genetic Resources, Hellenic Agricultural Organization Demeter, \\ 57001 Thermi, Greece \\ 4 School of Agriculture, Aristotle University of Thessaloniki, 54124 Thessaloniki, Greece \\ 5 Department of Agricultural Development, Democritus University of Thrace, 68200 Orestiada, Greece \\ * Correspondence: lisaninou@gmail.com
}

Received: 30 June 2019; Accepted: 6 August 2019; Published: 8 August 2019

check for updates

\begin{abstract}
Landraces are a valuable source of genetic variability for breeders to develop high-yielding lentil varieties. Apart from productivity, simultaneous breeding for lentil seed nutritional quality is of paramount importance for wider lentil consumption. This work examined the indirect effect of single plant selection for high yield on important seed quality traits within three Greek lentil landraces ("Elassona" (EL), "Lefkada" (L), and "Evros" (EV)). The breeding methodology applied was proved to help either maintain or improve such characteristics in the high-yielding second-cycle lines (SLs) selected. Compared to the parental landrace "Elassona", the high-yielding lines showed increased crude fiber by $30-110 \%$; the line 2-SL-EL-6 had higher starch content by $3.9 \%$ and reduced cooking time by $6.67 \mathrm{~min}$, while the 2-SL-EL-10 line had higher crude fiber by 73\%. In the case of "Lefkada", the high-yielding lines selected maintained the protein content present in the parental landrace, apart from the 2-SL-L-1 where a decrease by 5\% was recorded; however, most of them showed increased crude fiber (5.59-7.52\%) in comparison with the parental landrace $(4.65 \%)$. Finally, in relation to the "Evros" parental landrace, the 2-SL-EV-3 and 2-SL-EV-4 showed higher crude fiber and reduced cooking time. This study provides evidence that proper management of genetic variability could improve productivity without compromising or sometimes improving some seed quality traits.
\end{abstract}

Keywords: lentil landrace; seed quality; physicochemical characteristics; cooking time; protein; starch; crude fiber; breeding

\section{Introduction}

Lentil (Lens culinaris Medik.) is probably the oldest grain legume to be domesticated [1] and one of the most important pulse crops worldwide due its nutritional characteristics. It is considered an excellent source of complex carbohydrates, protein, minerals, vitamins, and dietary fibers [2,3]. Lentil is a highly nutritious legume both as human food and animal feed, and the chemical composition of the seeds is affected by genetic and environmental factors [4]. Despite its agronomic and nutritional value, lentil seed production remained at low levels and attracted much less attention by plant breeders than cereal grains until recently [4]. Currently, the extensive recognition of lentil's health benefits resulted in breeding varieties that are more productive and nutritious $[4,5]$. 
It is widely accepted that genotype affects lentil chemical properties considerably [3,6]; thus, to identify lentil genotype variability for seed quality traits would be valuable for the improvement of lentil quality and the production of varieties with high nutrition value, such as high protein content $[7,8]$. Wang and Daun [6] reported significant variability in protein content ranging from $24.3 \%$ to $30.2 \%$, whereas Wang et al. [9], who found crude protein content values between 251.5 and $292.5 \mathrm{~g} / \mathrm{kg}$ dry matter, concluded that cultivar moreover had a considerable impact on other nutritious constituents like starch, ash, and soluble dietary fiber. Starch content constitutes the highest proportion of the seed in lentil and was recorded to range between 35 and 53\% [10], 49 and 65\% [11], and 41 and $49 \%$ [12], while, in the case of ash content, values were recorded ranging from $2.13-3.42 \%$ [13] and $2.3-3.5 \%$ [2]. As for dietary fiber, which includes the plant-cell skeletal remains that are resistant to digestion [14], Huisman and van der Poel [15] and Hulse [16] found that lentil decorticated seeds contained $0.9 \mathrm{~g}$ of crude fiber/100 g, and other researchers reported values between $3.8 \%$ and $6 \%$ of dry seed weight [13]. Interestingly, Sulieman [17] reported that cultivars with high starch content generally had lower lipid content. Regarding lipid content, Devos [10] identified a range from 1-2\%, while, in other studies, values were documented ranging from $0.5-2.8 \%$ and $1-1.3 \%$ [2]. More specifically for fat, according to Adsule et al. [18] and Muehlbauer et al. [19], lentil seeds contain approximately $0.6 \mathrm{~g}$ of fat $/ 100 \mathrm{~g}$ of dried seeds, whereas Hulse [16] found concentrations of $1.8 \mathrm{~g}$ of fat $/ 100 \mathrm{~g}$ of decorticated lentil seeds.

Apart from seed chemical composition, genetic variability was documented for physicochemical characteristics, seed size, and seed processing (soaking, cooking, and dehulling). Cooking quality is connected with cooking time, which affects nutrient and anti-nutrient contents $[2,9,13]$. The seed coat of pulses is often indigestible and may have a bitter taste; thus, it could affect cooking quality and eventually consumption [3]. Seed coat and hot water ability to penetrate the cotyledon are genetically controlled and, thus, cause variability in cooking time in lentil varieties [20-22]. The cooking time of Turkish lentil varieties was recorded between 15.2 and $23.9 \mathrm{~min}$ [13]; Jood et al. [11] reported values ranging between 38 and $43 \mathrm{~min}$, while Vandenberg [23] recorded cooking times varying from 15 to $20 \mathrm{~min}$. Another parameter is water retention, i.e., the ability of food material to hold water against gravity, defined as "water absorption" [24]. Lentil genotype affected seed hydration capacity that ranged from 0.028-0.053 g/seed according to Özer and Kaya [13], while other researchers reported a range of 0.019-0.023 g/per seed in different lentil varieties [11]. Finally, significant differences among lentil varieties were documented in physical properties and morphological characteristics, such as 1000 -grain weight and size values $[13,21]$.

Seed cooking quality is one of the most important factors for the utilization of lentil as food because it is generally consumed in its cooked form. This characteristic is associated with the ease and cost of food preparation [3]; thus, emphasis should be put on improving or maintaining it. Selection for the improvement of seed quality characteristics is possible in lentil since genetic variability was recorded for total starch, protein, 1000-seed weight, seed color, and other quality characteristics [25]. Landraces present a valuable gene pool for a breeder to develop elite lines and varieties [26,27]. Vlachostergios et al. [28] applied intense single-plant selection among widely spaced individual plants in three lentil landraces aiming at second-generation sister lines of high yielding potential. When evaluated at farming density, the derived lines had mean grain yields $8 \%, 10 \%$, and $20 \%$ higher compared to their respective ancestors [28]. The above findings support the view that the development of pure line cultivars that fully meet the needs of sustainable agriculture is possible [29] and, at the same time, ensures optimum use of resources across variable conditions [30]. The main criterion for the selection within the Greek landraces was the yielding potential [28]; however, seed quality is an important parameter for lentil consumption; thus, the effect of the breeding method applied to the seed quality characteristics should be evaluated. The aim of the current study was to investigate the indirect effect of intense breeding for high yield on seed quality characteristics, as well as level of variability for these traits among the derived second-generation sister lines. 


\section{Materials and Methods}

\subsection{Genetic Material}

For the study, 30 second-generation single-plant sister lines were used, obtained via the honeycomb breeding methodology [29]. Selection was applied within three lentil landraces grown under a nil-competition regime, i.e., individual plants were widely spaced to preclude any plant-to-plant interference for inputs. In brief, within each landrace, selection of 30 out of 1000 initially established plants led to first-generation sister lines. Then, evaluation of the first-generation sister lines at the nil-competition regime at three locations followed by single-plant selection within the outstanding lines led to the development of 20 second-generation sister lines, which were further evaluated according to the nil-competition methodology at three locations. Finally, the most promising lines were tested under the common farming density at five environments to verify their yield superiority over their respective parental landrace. A detailed description of the breeding procedure is provided by Vlachostergios et al. [28], as presented in Figure 1.

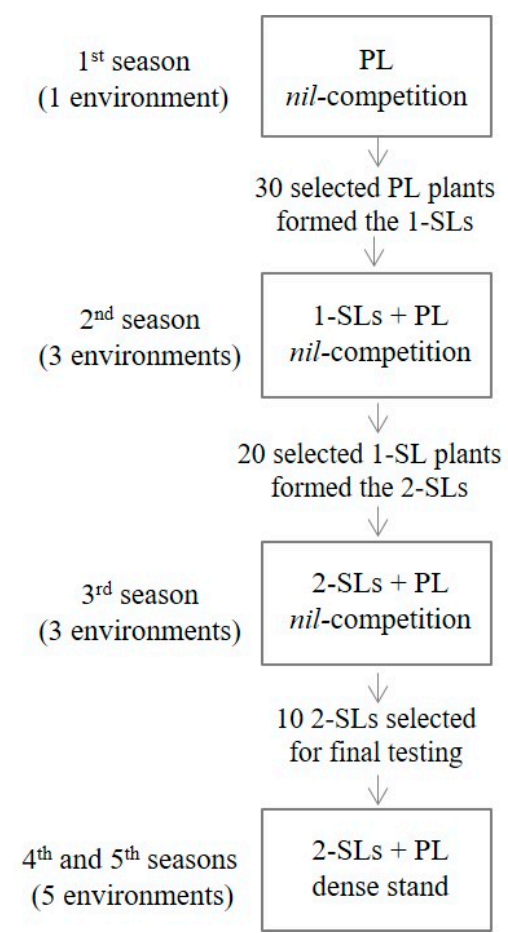

Figure 1. A line diagram of the breeding scheme including single-plant selection at the nil-competition regime within the parental landrace (PL) and the sister lines (SLs), with final SL evaluation at a dense stand (adapted from Vlachostergios et al. [28]). The seed material studied in the current work originated from one of the above final trials at a dense stand.

Seed material for quality analysis for the present study originated from one of the above final trials at farming conditions. That trial was established at the farm of the Fodder Crops and Pastures Institute in Larissa (39 $36^{\prime}$ north (N), 22 $2^{\circ} 5^{\prime}$ east (E), 74 meters above sea level (masl)) during 2015 under rain-fed conditions without any application of chemicals and fertilizers, while weeds were handled by hand-weeding as described by Vlachostergios et al. [28]. The experimental design was a randomized complete block design with three replications. The second-generation lines included in the trial were (i) 10 from the landrace "Elassona" (EL), coded as "2-SL-EL", (ii) 10 from the landrace "Lefkada" (L) coded as "2-SL-L", and (iii) seven from the landrace "Evros" (EV) coded as "2-SL-EV", whereas (iv) the three parental landraces (PL) were also included. 


\subsection{Agronomic and Morphological Seed Characteristics}

The 1000-seed weight in grams was determined by counting 200 lentil seeds using an electronic seed counter and scales. The results are expressed as the mean of duplicate measurements. Seed width and thickness were measured in $\mathrm{mm}$ for 20 seeds per plot. The seed yield as presented by Vlachostergios et al. [28] is provided in relation with the seed quality traits.

\subsection{Seed Coat Color}

The seed coat color of intact lentil seeds was recorded by a Minolta CR-410 chroma meter (Minolta, Osaka, Japan) using the granular material attachment CR-A50. Data were collected for $\mathrm{L}^{*}=$ lightness ranging from 0 (black) to 100 (white), $\mathrm{a}^{*}=$ redness/greenness, $\mathrm{b}^{*}=$ yellowness/blueness, $\mathrm{C}^{*}=$ chroma, and $\mathrm{H}=$ hue. A white porcelain reference plate $(Y=93.66, X=0.3150$, and $y=0.3217)$ supplied with the instrument was used for calibration. All color parameters for each sample were the instrument average of three independent measurements.

\subsection{Physicochemical Characteristics}

Seed coat percentage was determined for 20 seeds per plot as the weight ratio between coat and cotyledons expressed in percentage after removing the seed coat from the cotyledons, having soaked and kept the seeds for $24 \mathrm{~h}$ at $105^{\circ} \mathrm{C}$.

The hydration increase of lentil seeds was calculated as the percentage increase in mass of lentils soaked in distilled water for 12 and $24 \mathrm{~h}$. Hydration capacity expressed as hydration capacity per seed was determined by dividing the mass gained by the seeds either in 12 or $24 \mathrm{~h}$ by the number of seeds present in the sample [31]. All tests were carried out in duplicate.

Cooking time was estimated according to the method described by Iliadis [32]. Twenty grams of lentil seeds from each plot were added in $200 \mathrm{~mL}$ of distilled water in 250-mL conical flasks placed in a $100{ }^{\circ} \mathrm{C}$ water bath. After $20 \mathrm{~min}$ of initial cooking, samples of 10 seeds were taken from each flask at 5-min intervals. Needle intrusion depth was measured using a penetrometer (Sur PNR-6, Berlin Germany) for loading of $50 \mathrm{~g}$ and gravity of $0.2 \mathrm{sec}$. Seeds were considered cooked when the penetration value was $4 \mathrm{~mm}$.

\subsection{Nutritional Quality Seed Traits}

The nutritional quality traits were determined in duplicate finely ground samples. Seeds were ground in a Cyclotec mill to pass a 1.0-mm screen. The mineral ash percentage calculated on dry weight basis, total lipids, crude fiber, and total protein content (measured with the Kjeldhal method, $\mathrm{N} \times 6.25$ ) were determined using official methods [33]. Carbohydrates were determined by the difference.

\subsection{Statistical Analysis}

Analysis of variance (ANOVA) was conducted for randomized complete block design. The significance level of all hypotheses tested was pre-set at $p<0.05$, using the Tukey test $(p<0.05)$. Pearson correlation coefficients were also calculated for all traits. All statistical analyses were performed using the SPSS software package (ver. 18. SPSS Inc., Chicago, IL, USA).

\section{Results}

The recorded values of the seed quality characteristics for the three parental landraces and second-generation high-yielding sister lines [28] revealed variability in several traits. The effect of the selection for high seed yield on important seed quality characteristics on each of the three Greek landraces is present below in detail. 


\section{1. "Elassona" Second-Generation Sister Lines}

Concerning the EL lentil landrace, the protein, ash, lipids, 1000-seed weight, seed diameter and thickness, seed coat percentage, and hydration index were not affected by the selection for high yield (Tables 1 and 2). Similarly, seed coat color parameters $\left(\mathrm{L}^{*}, \mathrm{a}^{*}, \mathrm{~b}^{*}, \mathrm{C}^{*}\right.$, and $\left.\mathrm{H}\right)$ and hydration parameters were not affected (data not shown). However, the crude fiber values of the selected genotypes differed from the EL parental landrace and ranged from $3.21 \%$ to $6.94 \%$. In five selections, significant variability was found, and an increase from $30 \%$ to $110 \%$ was recorded in comparison with the parental landrace. The high-yielding selection 6 (2-SL-EL-6) had similar crude fiber concentration with the original genotype, whereas the high-yielding selection 10 (2-SL-EL-10) showed increased crude fiber by $73 \%$ in comparison with the EL parental landrace (Table 1).

Table 1. Seed yield and nutritional quality seed traits of the parental landrace "Elassona" (EL-PL) and second-generation sister lines (SLs) originating from "Elassona" (EL).

\begin{tabular}{ccccccc}
\hline $\begin{array}{c}\text { Lentil } \\
\text { Genotypes }\end{array}$ & $\begin{array}{c}\text { Seed Yield } \\
\mathbf{( k g} / \mathbf{h a})\end{array}$ & $\begin{array}{c}\text { Protein } \\
\mathbf{( \% )}\end{array}$ & $\begin{array}{c}\text { Ash } \\
\mathbf{( \% )}\end{array}$ & $\begin{array}{c}\text { Crude Fiber } \\
\mathbf{( \% )}\end{array}$ & $\begin{array}{c}\text { Lipids } \\
\mathbf{( \% )}\end{array}$ & $\begin{array}{c}\text { Starch } \\
\mathbf{( \% )}\end{array}$ \\
\hline EL-PL & 1124 & 28.09 & 2.65 & 3.26 & 1.06 & 53.58 \\
2-SL-EL-1 & 1278 & 26.80 & 3.05 & $4.81 * *$ & 0.77 & 53.12 \\
2-SL-EL-2 & 1170 & 27.30 & 3.02 & 3.35 & 0.63 & 54.47 \\
2-SL-EL-3 & 1038 & 27.49 & 3.02 & $4.69 * *$ & 0.72 & 52.66 \\
2-SL-EL-4 & 1196 & 26.88 & 2.88 & $6.94 * *$ & 0.48 & $51.12 *$ \\
2-SL-EL-5 & 1225 & 28.05 & 3.10 & $3.93 *$ & 0.83 & 52.86 \\
2-SL-EL-6 & $1387^{*}$ & 26.24 & 2.88 & 3.21 & 0.69 & $55.67 * *$ \\
2-SL-EL-7 & 1059 & 27.85 & 2.92 & 3.62 & 0.83 & 53.29 \\
2-SL-EL-8 & 1210 & 27.30 & 3.16 & 3.76 & 0.84 & 53.57 \\
2-SL-EL-9 & 1265 & 27.28 & 3.11 & 3.92 & 0.79 & 53.55 \\
2-SL-EL-10 & $1352 *$ & 27.66 & 2.99 & $5.65 *$ & 0.72 & $52.35 *$ \\
\hline$*{ }^{* *}$ Significant differences at the 0.05 and 0.01 levels of probability.
\end{tabular}

Table 2. Agronomical, morphological, seed coat $\%$, hydration index, and cooking time of the parental landrace "Elassona" and second-generation sister lines originating from "Elassona".

\begin{tabular}{|c|c|c|c|c|c|c|c|}
\hline $\begin{array}{c}\text { Lentil } \\
\text { Genotypes }\end{array}$ & $\begin{array}{l}\text { 1000-Seed } \\
\text { Weight (g) }\end{array}$ & $\begin{array}{l}\text { Diameter } \\
(\mathrm{mm})\end{array}$ & $\begin{array}{l}\text { Thickness } \\
\text { (mm) }\end{array}$ & $\begin{array}{c}\text { Seed Coat } \\
\text { Percentage }(\%)\end{array}$ & $\begin{array}{l}\text { Hydration } \\
\text { Index (12 h) }\end{array}$ & $\begin{array}{l}\text { Hydration } \\
\text { Index }(24 \mathrm{~h})\end{array}$ & $\begin{array}{l}\text { Cooking } \\
\text { Time (min) }\end{array}$ \\
\hline EL-PL & 32.90 & 4.57 & 2.51 & 8.01 & 0.83 & 0.90 & 41.67 \\
\hline 2-SL-EL-1 & 35.13 & 4.79 & 2.51 & 7.73 & 0.83 & 0.93 & $33.33 * *$ \\
\hline 2-SL-EL-2 & 40.33 & 5.00 & 2.56 & 7.77 & 0.84 & 0.94 & 38.33 \\
\hline 2-SL-EL-3 & 37.17 & 4.79 & 2.64 & 7.89 & 0.83 & 0.92 & 36.67 * \\
\hline 2-SL-EL-4 & 36.20 & 4.80 & 2.55 & 7.85 & 0.84 & 0.94 & 43.33 \\
\hline 2-SL-EL-5 & 29.07 & 4.28 & 2.50 & 7.99 & 0.85 & 0.96 & $31.67^{* *}$ \\
\hline 2-SL-EL-6 & 34.33 & 4.78 & 2.57 & 7.89 & 0.83 & 0.93 & $35.00 * *$ \\
\hline 2-SL-EL-7 & 36.20 & 4.77 & 2.53 & 7.40 & 0.86 & 0.95 & $35.00 * *$ \\
\hline 2-SL-EL-8 & 36.03 & 4.73 & 2.58 & 8.02 & 0.77 & 0.91 & 43.33 \\
\hline 2-SL-EL-9 & 34.70 & 4.59 & 2.59 & 8.00 & 0.86 & 0.93 & $35.00 * *$ \\
\hline 2-SL-EL-10 & 34.30 & 4.43 & 2.56 & 7.52 & 0.82 & 0.91 & 36.67 \\
\hline
\end{tabular}

The starch was also affected by the selection for high yield and ranged from $51.12 \%$ to $55.67 \%$. It is noteworthy that, in selection 6 (2-SL-EL-6), the starch content increased by 3.9\%. However, in two selections (2-SL-EL-4 and 2-SL-EL-10), inferior values were observed, and the remaining high-yielding sister lines showed no significant differences in starch concentration (Table 1). Cooking time connected with the consumption value of lentils varied from 31.67 to 43.33 min (Table 2). Remarkably, the high-seed-yielding selection 6 had a reduced cooking time by $6.67 \mathrm{~min}$ compared to the EL parental landrace.

In general, the protein content and seed quality characteristics of the high-yielding lines selected were not affected by the breeding methodology, since no differences were recorded in comparison with the parental landrace. However, the high-yielding selection 6 had higher starch content and shorter cooking time, while the high-yielding selection 10 was richer in crude fiber (Tables 1 and 2). 


\section{2. "Lefkada" Second-Generation Sister Lines}

The $\mathrm{L}$ lentil landrace and the high-yielding second-generation sister lines showed variability for protein $(26.8 \%-28.3 \%)$, crude fiber $(3.89 \%-7.52 \%)$, and starch content $(48.55 \%-53.9 \%)$ (Table 3$)$.

Table 3. Seed yield and nutritional quality traits of the parental landrace "Lefkada" (L-PL) and second-generation sister lines originating from "Lefkada".

\begin{tabular}{ccccccc}
\hline $\begin{array}{c}\text { Lentil } \\
\text { Genotypes }\end{array}$ & $\begin{array}{c}\text { Seed Yield } \\
\mathbf{( k g / h a )}\end{array}$ & $\begin{array}{c}\text { Protein } \\
\mathbf{( \% )}\end{array}$ & $\begin{array}{c}\text { Ash } \\
\mathbf{( \% )}\end{array}$ & $\begin{array}{c}\text { Crude fiber } \\
\mathbf{( \% )}\end{array}$ & $\begin{array}{c}\text { Lipids } \\
\mathbf{( \% )}\end{array}$ & $\begin{array}{c}\text { Starch } \\
\mathbf{( \% )}\end{array}$ \\
\hline L-PL & 1136 & 28.33 & 2.72 & 4.65 & 0.92 & 51.67 \\
2-SL-L-1 & $1455^{* *}$ & $26.82^{*}$ & 3.07 & $5.66^{* *}$ & 0.67 & 52.36 \\
2-SL-L-2 & $1343^{* *}$ & 28.90 & 3.13 & $6.16^{* *}$ & 0.73 & $49.39 *$ \\
2-SL-L-3 & $1379^{* *}$ & 28.30 & $3.24^{*}$ & 5.05 & 0.88 & 51.48 \\
2-SL-L-4 & $1395^{* *}$ & 27.42 & 3.50 & $7.30^{* *}$ & 0.68 & 49.65 \\
2-SL-L-5 & 1297 & $27.03^{*}$ & 2.95 & $5.39 *$ & $0.53 *$ & 52.89 \\
2-SL-L-6 & $1446^{* *}$ & 27.72 & 2.67 & $7.52^{* *}$ & 0.58 & 50.09 \\
2-SL-L-7 & 1278 & 28.10 & 2.76 & $5.59 *$ & 0.93 & 51.17 \\
2-SL-L-8 & 1259 & 27.43 & 2.80 & $3.89 *$ & 0.70 & $53.90 *$ \\
2-SL-L-9 & $1357^{* *}$ & 27.80 & 2.64 & $6.66^{* *}$ & 0.74 & $48.55 * *$ \\
2-SL-L-10 & $1507^{* *}$ & 27.31 & 3.28 & 5.01 & 0.77 & 52.32 \\
\hline
\end{tabular}

The selection for high yield in the $\mathrm{L}$ lentil landrace did not affect the protein content in the majority of the high-yielding sister lines compared with the parental landrace. Among the high-yielding selections, only 2-SL-L-1, which had the second highest yield (1455 kg/ha), showed a decrease in protein content by $5 \%$ (Table 4). However, the selection for high yield was accompanied by an increase in crude fiber for eight selections in comparison with the parental landrace (Table 3).

Table 4. Agronomical, morphological, seed coat \%, hydration index, and cooking time of the parental landrace "Lefkada" and second-generation sister lines originating from "Lefkada".

\begin{tabular}{|c|c|c|c|c|c|c|c|}
\hline $\begin{array}{c}\text { Lentil } \\
\text { Genotypes }\end{array}$ & $\begin{array}{l}\text { 1000-Seed } \\
\text { Weight (g) }\end{array}$ & $\begin{array}{l}\text { Diameter } \\
(\mathrm{mm})\end{array}$ & $\begin{array}{c}\text { Thickness } \\
(\mathrm{mm})\end{array}$ & $\begin{array}{c}\text { Seed Coat } \\
\text { Percentage }(\%)\end{array}$ & $\begin{array}{l}\text { Hydration } \\
\text { Index (12 h) }\end{array}$ & $\begin{array}{c}\text { Hydration } \\
\text { Index (24 h) }\end{array}$ & $\begin{array}{l}\text { Cooking Time } \\
\text { (min) }\end{array}$ \\
\hline L-PL & 30.27 & 4.34 & 2.56 & 8.55 & 0.83 & 0.92 & 33.33 \\
\hline 2-SL-L-1 & 37.90 & 4.72 & 2.62 & 7.70 & 0.85 & 0.94 & 33.33 \\
\hline 2-SL-L-2 & 34.83 & 4.74 & 2.54 & 8.20 & 0.80 & 0.95 & 26.67 \\
\hline 2-SL-L-3 & 33.20 & 4.54 & 2.53 & 8.10 & 0.84 & 0.95 & 28.33 \\
\hline 2-SL-L-4 & 36.27 & 4.61 & 2.48 & 7.81 & 0.82 & 0.92 & 31.67 \\
\hline 2-SL-L-5 & 36.30 & 4.78 & 2.60 & 7.96 & 0.83 & 0.93 & 30.00 \\
\hline 2-SL-L-6 & 38.27 & 4.90 & 2.55 & 7.89 & 0.83 & 0.92 & 31.67 \\
\hline 2-SL-L-7 & 32.17 & 4.37 & 2.52 & 7.98 & 0.83 & 0.94 & 26.67 \\
\hline 2-SL-L-8 & 35.10 & 4.63 & 2.49 & 8.16 & 0.80 & 0.90 & 30.00 \\
\hline 2-SL-L-9 & 36.20 & 4.80 & 2.54 & 8.15 & 0.88 & 0.97 & 30.00 \\
\hline 2-SL-L-10 & 31.85 & 4.22 & 2.55 & 7.96 & 0.81 & 0.89 & 35.00 \\
\hline
\end{tabular}

Regarding starch content, the selection 2-SL-L-8 had higher starch content in comparison with the parental landrace, although it was not differentiated for seed yield (Table 3). The selections 2-SL-L-2 and 2-SL-L-9 showed reduced values by $\sim 5 \%$, while the other five high-yielding selections did not differ for starch content (Table 3). The breeding scheme applied aimed at seed yield increase, and seven out of the total 10 second-generation sister lines had higher yield. At the same time, the methodology based on a single-plant selection sustained the other seed quality characteristics in the "Lefkada" landrace. The 1000-seed weight, seed diameter and thickness, seed coat percentage, hydration index, and cooking time remained constant in comparison with the "Lefkada" parental landrace (Table 4). Also, the seed coat color parameters $\left(\mathrm{L}^{*}, \mathrm{a}^{*}, \mathrm{~b}^{*}, \mathrm{C}^{*}\right.$, and $\left.\mathrm{H}\right)$ and the hydration increases, coefficients, and capacities were not altered (data not shown). 


\section{3. "Evros" Second-Generation Sister Lines}

Concerning the EV lentil landrace, the protein, ash, lipids, 1000-seed weight, seed diameter and thickness, seed coat percentage, and hydration index were not affected by the selection for high yield (Tables 5 and 6). Also, no effect on seed coat color parameters $\left(\mathrm{L}^{*}, \mathrm{a}^{*}, \mathrm{~b}^{*}, \mathrm{C}^{*}\right.$, and $\left.\mathrm{H}\right)$ and hydration increases, coefficients, and capacities was found (data not shown). Nevertheless, the seed characteristics of the EV second-generation sister lines showed variability for crude fiber, starch content, and cooking time ranging from $3.12 \%$ to $5.62 \%, 49.93 \%$ to $54.06 \%$, and 31.67 to $41.67 \mathrm{~min}$, respectively (Table 5). Noticeably, six sister lines selected had increased crude fiber in comparison with the parental landrace "Evros", and two of them (2-SL-EV-3 and 2-SL-EV-4) had significantly higher seed yield (Table 5). The starch content was decreased by $4.7 \%$ in the high-seed-yielding selection 2-SL-EV-3, while it was not altered in the high-seed-yielding selection 2-SL-EV-4 compared to the parental landrace EV. Three second-generation sister lines, including the two highest-yielding 2-SL-EV-3 and 2-SL-EV-4 selected lines, showed a highly significant lower cooking time by almost $10 \mathrm{~min}$ compared to the original genotype.

Table 5. Seed yield and nutritional quality traits of the parental landrace "Evros" (EV-PL) and second-generation sister lines originating from "Evros".

\begin{tabular}{ccccccc}
\hline $\begin{array}{c}\text { Lentil } \\
\text { Genotypes }\end{array}$ & $\begin{array}{c}\text { Seed Yield } \\
\mathbf{( k g} / \mathbf{h a})\end{array}$ & $\begin{array}{c}\text { Protein } \\
\mathbf{( \% )}\end{array}$ & $\begin{array}{c}\text { Ash } \\
\mathbf{( \% )}\end{array}$ & $\begin{array}{c}\text { Crude Fiber } \\
\mathbf{( \% )}\end{array}$ & $\begin{array}{c}\text { Lipids } \\
\mathbf{( \% )}\end{array}$ & $\begin{array}{c}\text { Starch } \\
\mathbf{( \% )}\end{array}$ \\
\hline EV-PL & 1100 & 28.19 & 2.62 & 3.12 & 0.86 & 53.57 \\
2-SL-EV-1 & 992 & 27.24 & 2.62 & 3.82 & 0.93 & 54.06 \\
2-SL-EV-2 & 1194 & 29.44 & 2.64 & $5.62^{* *}$ & 0.85 & $49.93^{* *}$ \\
2-SL-EV-3 & $1455^{* *}$ & 29.02 & 2.67 & $4.86^{* *}$ & 0.96 & $51.07 *$ \\
2-SL-EV-4 & $1412^{* *}$ & 27.64 & 2.74 & $4.91^{* *}$ & 0.88 & 52.37 \\
2-SL-EV-5 & 1188 & 27.37 & 2.51 & $4.08^{*}$ & 1.11 & 53.46 \\
2-SL-EV-6 & 1048 & 27.33 & 2.50 & $5.25^{* *}$ & 0.89 & 52.52 \\
2-SL-EV-7 & 1187 & 27.94 & 2.61 & $4.41^{* *}$ & 0.90 & 52.61 \\
\hline
\end{tabular}

$*{ }^{* *}$ Significant differences at the 0.05 and 0.01 levels of probability.

Table 6. Agronomical, morphological, seed coat $\%$, hydration index, and cooking time of the parental landrace "Evros" and second-generation sister lines originating from "Evros".

\begin{tabular}{|c|c|c|c|c|c|c|c|}
\hline $\begin{array}{c}\text { Lentil } \\
\text { Genotypes }\end{array}$ & $\begin{array}{l}\text { 1000-Seed } \\
\text { Weight (g) }\end{array}$ & $\begin{array}{l}\text { Diameter } \\
(\mathrm{mm})\end{array}$ & $\begin{array}{l}\text { Thickness } \\
(\mathrm{mm})\end{array}$ & $\begin{array}{c}\text { Seed Coat } \\
\text { Percentage }(\%)\end{array}$ & $\begin{array}{l}\text { Hydration } \\
\text { Index (12 h) }\end{array}$ & $\begin{array}{l}\text { Hydration } \\
\text { Index }(24 \mathrm{~h})\end{array}$ & $\begin{array}{l}\text { Cooking } \\
\text { Time (min) }\end{array}$ \\
\hline EV-PL & 35.40 & 4.62 & 2.54 & 7.94 & 0.85 & 0.95 & 41.67 \\
\hline 2-SL-EV-1 & 39.37 & 5.03 & 2.56 & 7.88 & 0.86 & 0.94 & 40.00 \\
\hline 2-SL-EV-2 & 36.73 & 4.84 & 2.58 & 7.73 & 0.86 & 0.95 & $31.67^{* *}$ \\
\hline 2-SL-EV-3 & 34.40 & 4.53 & 2.58 & 7.72 & 0.84 & 0.93 & $31.67^{* *}$ \\
\hline 2-SL-EV-4 & 33.17 & 4.33 & 2.59 & 7.60 & 0.81 & 0.92 & $31.67^{* *}$ \\
\hline 2-SL-EV-5 & 35.27 & 4.80 & 2.51 & 8.04 & 0.84 & 0.94 & 40.00 \\
\hline 2-SL-EV-6 & 36.57 & 4.79 & 2.62 & 7.98 & 0.82 & 0.92 & 36.67 \\
\hline 2-SL-EV-7 & 35.67 & 4.62 & 2.54 & 8.13 & 0.85 & 0.94 & 35.00 \\
\hline
\end{tabular}

The application of the breeding scheme as described by Vlachostergios et al. [28] for two selection cycles allowed the selection for higher yield without compromising important seed quality characteristics for the majority of the genotypes selected compared to their respective parental landraces. The variation and descriptive statistics for important parameters like nutritional quality traits (Table S1, Supplementary Materials), agronomical, morphological, and seed coat characteristics (Table S2, Supplementary Materials), and hydration parameters and cooking time (Table S3, Supplementary Materials) were not differentiated in comparison with the parental landraces.

Finally, the correlation between agronomical, morphological, physicochemical, and nutritional quality traits and seed coat characteristics was estimated. A negative correlation was found between starch content and crude fiber $\left(\mathrm{r}_{\text {st.-cf }}=-0.856^{* *}\right)$, whereas seed diameter and 1000-seed weight were positively correlated $\left(\mathrm{r}_{\mathrm{sd}-1000 \mathrm{sw}}=0.881^{* *}\right)$ (Table 7). Also, a positive correlation was found between $\mathrm{L}^{*}$ (lightness) and 1000-seed weight, $\mathrm{b}^{*}$ (yellowness/blueness), $\mathrm{C}^{*}$ (chroma), $\mathrm{H}$ (hue) $\left(\mathrm{r}_{\mathrm{L}-1000 \mathrm{SW}}=\right.$ $0.690^{* *}, \mathrm{r}_{\mathrm{L}-\mathrm{b}}=0.855^{* *}, \mathrm{r}_{\mathrm{L}-\mathrm{C}}=0.851^{* *}$, and $\mathrm{r}_{\mathrm{L}-\mathrm{H}}=0.738^{* *}$, respectively), between $\mathrm{H}$ (hue) and $\mathrm{b}^{*}$ (yellowness/blueness) and $\mathrm{C}^{*}$ (chroma) $\left(\mathrm{r}_{\mathrm{H}-\mathrm{b}}=0.879^{* *}\right.$ and $\mathrm{r}_{\mathrm{H}-\mathrm{C}}=0.788^{* *}$, respectively), and between $\mathrm{C}^{*}$ (chroma) and $\mathrm{b}^{*}$ (yellowness/blueness) $\left(\mathrm{r}_{\mathrm{C}-\mathrm{b}}=0.985^{* *}\right)$. 
Table 7. Correlation between agronomical, morphological, physicochemical nutritional quality traits and seed coat characteristics.

\begin{tabular}{|c|c|c|c|c|c|c|c|c|c|c|c|c|c|c|c|c|c|}
\hline & Yield & Protein & Ash & $\begin{array}{l}\text { Crude } \\
\text { Fiber }\end{array}$ & Lipids & Starch & $\begin{array}{l}\text { 1000-Seed } \\
\text { Weight }\end{array}$ & Diameter & Thickness & $\mathrm{L}^{*}$ & $a^{*}$ & $\mathbf{b}^{*}$ & $\mathrm{C}^{*}$ & H & $\begin{array}{l}\text { Seed } \\
\text { Coat }\end{array}$ & $\begin{array}{c}\text { Hydration } \\
\text { Increase (24 h) }\end{array}$ & $\begin{array}{c}\text { Cooking } \\
\text { Time }\end{array}$ \\
\hline Yield & 1 & 0.005 & 0.359 & $0.468^{* *}$ & -0.318 & -0.377 & -0.180 & -0.326 & -0.024 & -0.052 & 0.214 & -0.314 & -0.290 & -0.281 & -0.145 & -0.129 & $-0.541^{* *}$ \\
\hline Protein & & 1 & -0.203 & 0.107 & $0.425^{*}$ & -0.538 & -0.307 & -0.313 & -0.132 & -0.204 & 0.220 & -0.048 & 0.012 & -0.217 & 0.161 & 0.226 & -0.349 \\
\hline Ash & & & 1 & 0.163 & $-\underset{*}{-0.446}$ & -0.064 & -0.062 & -0.148 & -0.091 & 0.080 & -0.010 & -0.161 & -0.175 & -0.099 & -0.145 & -0.050 & -0.211 \\
\hline Crude fiber & & & & 1 & $-\underset{* *}{-0.467}$ & $-\underset{* *}{-0.856}$ & 0.141 & 0.036 & 0.019 & 0.041 & 0.286 & -0.262 & -0.191 & -0.384 * & -0.034 & 0.095 & $-0.422 *$ \\
\hline $\begin{array}{l}\text { Lipids } \\
\text { Starch }\end{array}$ & & & & & 1 & $\begin{array}{c}0.151 \\
1\end{array}$ & $\begin{array}{c}-0.388^{*} \\
0.044\end{array}$ & $\begin{array}{c}-0.303 \\
0.102\end{array}$ & $\begin{array}{c}-0.190 \\
0.089\end{array}$ & $\begin{array}{l}-0.243 \\
0.127\end{array}$ & $\begin{array}{l}-0.088 \\
-0.360\end{array}$ & $\begin{array}{l}0.133 \\
0.273\end{array}$ & $\begin{array}{l}0.124 \\
0.188\end{array}$ & $\begin{array}{c}0.122 \\
0.452 *\end{array}$ & $\begin{array}{c}0.189 \\
-0.126\end{array}$ & $\begin{array}{l}-0.020 \\
-0.285\end{array}$ & $\begin{array}{c}0.132 \\
0.517 \text { ** }\end{array}$ \\
\hline $\begin{array}{c}\text { 1000-Seed } \\
\text { Weight }\end{array}$ & & & & & & & 1 & $0.881^{* *}$ & 0.301 & $\underset{* *}{0.690}$ & 0.017 & $0.583^{* *}$ & $0.600 * *$ & $0.458 *$ & -0.336 & 0.075 & 0.291 \\
\hline Diameter & & & & & & & & 1 & 0.158 & 0.586 & -0.028 & $0.542 * *$ & $0.547^{* *}$ & $0.446^{*}$ & -0.136 & 0.238 & 0.314 \\
\hline $\begin{array}{l}\text { Thickness } \\
\text { L }^{*}\end{array}$ & & & & & & & & & 1 & 0.032 & $\begin{array}{c}0.040 \\
-0.170\end{array}$ & $\begin{array}{c}0.042 \\
0.855 * *\end{array}$ & $\begin{array}{c}0.054 \\
0.851 * *\end{array}$ & $\begin{array}{c}0.006 \\
0.738 \text { ** }\end{array}$ & $\begin{array}{c}-0.179 \\
-0.503 * *\end{array}$ & -0.163 & 0.123 \\
\hline $\begin{array}{l}\mathrm{L}^{*} \\
\mathrm{a}^{*}\end{array}$ & & & & & & & & & & 1 & $\begin{array}{c}-0.170 \\
1\end{array}$ & $\begin{array}{l}0.855^{* *} \\
-0.221\end{array}$ & $\begin{array}{l}0.851^{* *} \\
-0.064\end{array}$ & $\begin{array}{c}0.738^{* *} \\
-0.605^{* *}\end{array}$ & $\begin{array}{c}-0.503 * * \\
0.032\end{array}$ & $\begin{array}{c}0.033 \\
-0.111\end{array}$ & $\begin{array}{c}0.157 \\
-0.066\end{array}$ \\
\hline$b^{*}$ & & & & & & & & & & & & 1 & $0.985 * *$ & 0.879 ** & $-439 *$ & 0.032 & 0.423 * \\
\hline$C^{*}$ & & & & & & & & & & & & & 1 & $0.788 * *$ & $-451 *$ & 0.016 & $0.417^{*}$ \\
\hline $\mathrm{H}$ & & & & & & & & & & & & & & 1 & $-378^{*}$ & 0.021 & 0.394 * \\
\hline Seed Coat & & & & & & & & & & & & & & & 1 & -0.015 & 0.134 \\
\hline $\begin{array}{l}\text { Hydration } \\
\text { Increase }(24 \mathrm{~h})\end{array}$ & & & & & & & & & & & & & & & & 1 & -0.225 \\
\hline $\begin{array}{l}\text { Increase }(24 \mathrm{~h}) \\
\text { Cooking time }\end{array}$ & & & & & & & & & & & & & & & & & 1 \\
\hline
\end{tabular}

${ }^{*}{ }^{* *}$ Significant differences at the 0.05 and 0.01 levels of probability. 


\section{Discussion}

Legumes could provide a sustainable solution for food production in terms of protein security. Concerning nutrition, landraces have higher seed protein content than modern varieties, as recorded in common bean [34]. In this sense, lentil landraces of good quality and high nutritional value that are adapted to local environmental conditions present a valuable source of genetic variability [28]. In this work, the selection of second-generation sister lines employed single-plant yield as the main selection criterion [28] without focusing on quality characteristics as a direct selection criterion. The scope of studying multiple quality characteristics was to investigate the indirect effect of this breeding scheme on seed quality. Variability regarding important seed quality traits and physicochemical characteristics was recorded for the three Greek lentil landraces and the second-generation sister lines examined. The main significant differences were identified in protein, crude fiber, starch content, and cooking time. Remarkably, some high-yielding selected lines displayed unaltered protein content and other important quality characteristics.

Iqbal et al. [35] stated that a major challenge in legume breeding is to increase productivity and nutritional quality; however, they recognized that quality evaluation requires screening both in the field and laboratory; thus, it is a time- and resource-consuming selection process. This method of single-plant selection for high seed yield under a nil-competition regime presents an approach that could minimize the time and resources required to improve productivity and maintain nutritional seed quality. Interestingly, the same methodology was also recommended as an effective agronomic practice to improve the sanitary status of lentil landraces seed stock during the seed propagation process, especially in case of seed-borne viruses that are transmitted by seed in high rate [36].

The protein concentration measured in the present study ranged from $26 \%$ to $28 \%$, which agrees with the values reported by Wang and Daun [6], who calculated a mean level of $27.2 \%$ in protein content. Likewise, protein contents reported in lentils were around 25\% [37], between $24.3 \%$ and $30.2 \%$ [12], and between 22.1 and $27.4 \%$ [38]. In this work, the protein content was unaltered in comparison with the parental landraces, even when the seed yield increased. Thus, direct selection for high yield in the EL landrace helped to maintain the seed protein content. A similar picture was drawn in the case of all the L high-yielding sister lines selected, apart from the 2-SL-L-1 that showed a decrease by $5 \%$. This was also the case for the protein content of the EV second-generation sister lines that did not differ compared to the parental landrace, even for the two selections with the highest yield (2-SL-EV-3 and 2-SL-EV-4). Such results are particularly promising since several researchers recorded a negative correlation between yield and protein content, which can be alleviated by breeding for both traits since sufficient variation for these coexists in legumes [39]. Iqbal et al. [35] suggested that an approach to exploit genotypic variability within legume populations is to select for increased yield and simultaneously aim to maintain a constant protein level. The breeding scheme of this study (Figure 1) [28] supplemented with the evaluation of quality traits could be proposed in alignment with the above recommendation.

In terms of crude fiber, it ranged from $3.12 \%$ to $7.52 \%$ for the three landraces and their second-generation sister lines studied; such levels are in agreement with those reported for Turkish lentil varieties (between 3.8\% and 6\%) [13]. In this study, the selection for high yield was accompanied by stable or increased crude fiber content in some selections originating from EL, L, and EV landraces. The crude fiber content increased (2-SL-EL-10) or remained stable (2-SL-EL-6) in two high-yielding selections from the EL landrace, while the crude fiber for the eight $L$ selections increased. Differences in fiber content among lentil cultivars exist due to genetic variability, suggesting a rationale for cultivar-based food labeling [40].

Regarding the starch content measurements in this study, a range from $48.55 \%$ to $55.67 \%$ was close to levels previously reported [38]; this parameter was affected in comparison with the parental landraces in some high-yielding selections of the EL, L, and EV landraces. Thus, the starch content in the two high-yielding EL selections was reduced (2-SL-L-2 and 2-SL-L-9), while the starch content of the EV selections decreased or remained constant in comparison with the EV parental landrace. 
Lentil genetic resources display significant variability for seed quality characteristics such as color, ranging from yellow to red-orange to green, brown, and black, as well as seed coat and cotyledon color [41]. Other quality traits that may be affected by cultivar are cooking quality, seed size, uniformity, and absence of split and discolored seeds [42]. We found that the intense breeding applied in three lentil landraces not only resulted in maintaining color and seed size unaltered compared with the parental landraces, but also resulted in improving cooking time in some of the lines selected. Jood et al. [11] reported that lentil cultivars differed in seed hydration capacity, and flat seeds with large surface area absorbed more water in comparison with those with narrow surface. A similar connection with seed size and water absorbance was identified also by Özer and Kaya [13], since flat lentil varieties with a large surface area revealed a more swollen appearance after water treatment, and varieties with narrow surface area absorbed less water per seed. In the current work, no differences were recorded for hydration capacity, seed size, and seed color, apart from cooking time which decreased, especially in the "Elassona" and "Evros" high-yielding selected lines by almost 6 and $10 \mathrm{~min}$, respectively.

Several important qualitative characteristics examined like average seed weight, protein content, and cooking quality showed high broad-sense heritability [21]. Plant breeders are interested mainly in traits having high heritability since they result in greater advance under selection. The values reported for seed weight of lentil were $62.8 \%$ [43], 87.0\% [44], 91.0\% [45], 98.0\% [21,46], and 99.0\% [47]. High broad-sense heritability was also found for protein content with a value of $84.0 \%$ [47] and cooking quality with $82.0 \%$ [21] and $98.0 \%$ [47]. Finally, moderate to high broad-sense heritability was found for lentil grain yield as reported by several researchers, ranging from $41.0 \%$ [45] to $48.0 \%$ [44], 59.7\% [43], $83.9 \%$ [46], and $96.0 \%$ [47].

Several researchers reported correlations between different seed quality characteristics in lentil genotypes. In this work, a negative correlation was found between starch content and crude fiber. A negative correlation was identified between starch and protein content [6,25], and also 1000-seed weight and protein concentration [25]. Similarly, a negative correlation was reported for seed yield and protein content, whereas cooking time and seed weight were positively correlated [21]. Seed yield was previously positively correlated to both cooking time and seed size, which affects cooking quality [21], a sign that seed size might be used to predict cooking time [47]. However, in this work, no correlation was found between cooking time and seed size, supporting the fact that the above might not be always be the case. A positive correlation was found between the 1000-seed weight and seed starch, total raffinose family oligosaccharides, and sucrose, while there was no correlation between the seed coat color and protein concentration [48]. Finally, in this work, while there was no correlation between the protein content and seed color, the seed diameter and 1000-seed weight were positively correlated, showing that the increase in seed weight is connected with an increase in seed diameter rather than its thickness.

Breeding strategies include the development of more productive varieties with favorable quality characteristics. In the case of lentil, research is underway for the development of improved plant types with high yielding ability and resistance to biotic and abiotic stress, while better nutrition quality is also a major breeding objective. So far, however, studies often focused on either yield gain or quality improvement rather than the influence of breeding techniques on such parameters combined. The procedure presented here provides the tools to exploit the natural genetic variability within landraces and develop, in a short time, pure-line varieties adaptable to a wide range of conditions that moreover display desirable characteristics [28]. It is recognized that yield is negatively correlated with protein content, and it is important to understand the relationship among different quality parameters for the selection of new cultivars with favorable quality characteristics [25]. There is evidence supported by this study that the proper management of genetic variability shows potential for the simultaneous increase of productivity and seed quality characteristics. There is need for more in-depth studies regarding the nutritional quality of this low-cost protein source and the influence of compounds such as fiber and related substances [49]. 
Even though huge genetic variation facilitates the selection of superior genotypes, and selection within a landrace is definitely one of the oldest plant breeding methods, the innovation brought about by the method presented here is that it leads toward the identification of a different class of genotypes in order to develop varieties in accordance with the main principles of sustainability. Based on the well-established negative relationship between genotype yield and competitive ability, breeding in conditions that ensure nil-competition instead of dense stand, and applying single-plant yield as the main selection criterion puts additional emphasis on highlighting the "weak competitor of high plant yield efficiency" ideotype [30]. The hypothesis of this approach is that genotypes characterized as "weak competitors" are exceptionally resilient to environmental forces that induce acquired intra-crop variation and, thus, could be employed to optimize resource use at crop level, while, at the same time, efficiency in resource use at single-plant level promotes stability due to better overall results in case of missing plants in the field [50].

\section{Conclusions}

The development of varieties combining high productivity and favorable nutritional characteristics constitutes a challenge in lentil breeding for increased consumption and widespread cultivation. The applied breeding scheme and selection for high yield based on plant performance at ultra-low density ensured in two cycles of selection that the seed quality characteristics of the landraces parental population remained unaltered. This could be an effective strategy to manipulate the existing genetic variability for seed quality and develop varieties combining higher seed yield and quality. According to the results of this work, the proposed breeding procedure favors the selection of high-yielding ideotypes that sustain qualitative characteristics. This innovative approach in breeding methodology provides evidence that breeding for high yield without degrading quality traits might be feasible.

Supplementary Materials: The following are available online at http://www.mdpi.com/2077-0472/9/8/175/s1, Table S1: Descriptive statistics of nutritional quality traits of second-generation sister lines originating from "Elassona", "Lefkada", and "Evros", Table S2: Descriptive statistics of agronomical, morphological, and seed coat characteristics of second-generation sister lines originating from "Elassona", "Lefkada", and "Evros", Table S3: Descriptive statistics of hydration increases, coefficients, capacities, and indices and cooking times of each parental landrace and second-generation sister lines originating from their respective parental landrace.

Author Contributions: Conceptualization, I.T.; data curation, F.P. and I.M.; formal analysis, I.M.; funding acquisition, I.T.; investigation, E.N.; methodology, F.P., D.N.V., and I.P.; project administration, I.T.; resources, E.N., A.K., C.P., and I.P.; writing-original draft, E.N., F.P., D.N.V., I.M., E.S., and I.T.; writing—review and editing, E.N., F.P., D.N.V., I.M., A.K., C.P., I.P., E.S., and I.T.

Funding: Research co-financed by the European Union (European Social Fund) and Greek national funds in the framework of the project "THALIS-Democritus University of Thrace-Selection for enhanced yield and tolerance to viral and vascular diseases within lentil landraces" through the Operational Program of the NSRF (National Strategic Reference Framework) "Education and lifelong learning investing in knowledge society".

Conflicts of Interest: The authors declare no conflict of interest.

\section{References}

1. Bahl, P.N.; Lal, S.; Sharma, B.M. An overview of the production and problems in South East Asia. In Lentil in South Asia, Proceedings of the Seminar on Lentils in South Asia, New Delhi, India, 11-15 March 1991; Erskine, W., Saxena, M.C., Eds.; ICARDA: Aleppo, Syria, 1993; pp. 1-10.

2. Wang, N.; Hatcher, D.W.; Toews, R.; Gawalko, E.J. Influence of cooking and dehulling on nutritional composition of several varieties of lentils (Lens culinaris). LWT Food Sci. Technol. 2009, 42, 842-848. [CrossRef]

3. Joshi, M.; Timilsena, Y.; Adhikari, B. Global production, processing and utilization of lentil: A review. J. Integr. Agric. 2017, 16, 2898-2913. [CrossRef]

4. Erskine, W.; Sarker, A. Lentil-Breeding. In Encyclopedia of Grain Science; Wrigley, C., Corke, H., Walker, C., Eds.; Elsevier: Amsterdam, The Netherlands, 2004; pp. 142-150.

5. Muehlbauer, F.J.; Kaiser, W.J.; Clement, S.L.; Summerfield, R.J. Production and Breeding of Lentil; Sparks, D.L., Ed.; Advances in Agronomy; Academic Press: San Diego, CA, USA, 1995; Volume 54, pp. 283-332. [CrossRef] 
6. Wang, N.; Daun, J.K. Effects of variety and crude protein content on nutrients and anti-nutrients in lentils (Lens culinaris). Food Chem. 2006, 95, 493-502. [CrossRef]

7. Savage, G.P. The composition and nutritive value of lentils (Lens culinaris). Nutr. Abstr. Rev. (Ser. A) 1988, 5, 320-343.

8. Burstin, J.; Gallardo, K.; Mir, R.R.; Varshney, R.K.; Duc, G. Improving protein content and nutritional quality. In Biology and Breeding of Food Legumes; Pratap, A., Kumar, J., Eds.; CAB International: Wallingford, UK, 2011.

9. Wang, N.; Hatcher, D.W.; Gawlko, E.J. Effect of variety and processing on nutrients and certain anti-nutrients in field peas (Pisum sativum). Food Chem. 2008, 111, 132-138. [CrossRef]

10. Devos, P. Nutritional value of lentils and chickpeas and changes during processing. In Herkes için Mercimek Sempozyumu; Bildiri Kitabi: Marmaris, Turkey, 1988; pp. 186-196.

11. Jood, S.; Bishnoi, S.; Sharma, A. Chemical analysis and physico-chemical properties of chickpea and lentil cultivars. Nahrung 1998, 42, 71-74. [CrossRef]

12. Wang, N.; Daun, J.K. The Chemical Composition and Nutritive Value of Canadian Pulses: Lentil (Lens culinaris); Canadian Grain Commission Grain Research Laboratory: Winnipeg, MB, Canada, 2004; pp. 8-18.

13. Özer, M.S.; Kaya, F. Physical, chemical and physicochemical properties of some lentil varieties grown in Turkey. J. Food Agric. Environ. 2010, 8, 610-613.

14. Jansen, G.R. A consideration of allowable fibre levels in weaning foods. Food Nutr. Bull. 1980, $2,38-47$. [CrossRef]

15. Huisman, J.; van der Poel, A.F.B. Expanding the Production and Use of Cool Season Food Legumes; Muehlbauer, F.J., Kaiser Kluwer, W.J., Eds.; Academic Publishers: Washington, DC, USA, 1994; pp. 53-77.

16. Hulse, J.H. Nature, composition and utilization of grain legumes. In ICRISAT. Uses of Tropical Grain Legumes: Proceedings of a Consultants' Meeting, ICRISAT Center, India, 27-30 March, 1989; ICRISAT: Patancheru, India, 1990; pp. 11-27.

17. Sulieman, M.A. Physicochemical and Structural Characterization of Germinated and Cooked Lentils (Lens culinaris Medic). Ph.D. Thesis, University of Khartoum, Khartoum, Sudan, October 2007.

18. Adsule, R.N.; Kadam, S.S.; Leung, H.K. Lentil. In Handbook of World Food Legumes: Nutritional Chemistry, Processing Technology, and Utilization; Salunkhe, D.K., Kadam, S.S., Eds.; CRC Press: Boca Raton, FL, USA, 1989; Volume 2, pp. 133-152.

19. Muehlbauer, F.J.; Cubero, J.I.; Summerfield, R.J. Lentil (Lens culinaris Medik.). In Grain Legume Crops; Summerfield, R.J., Roberts, E.H., Eds.; Collins, 8 Grafton Str: London, UK, 1985; pp. 266-311.

20. Singh, V.; Kherdekar, M.S.; Sharma, D.; Saxema, K.B. Cooking quality and chemical composition of some early, medium and late maturing cultivars of pigeon pea (Cajanus cajan L.). J. Food Sci. Technol. 1984, 2, 1367.

21. Erskine, W.; Williams, P.C.; Nakkoul, H. Genetic and Environmental Variation in the seed size, protein, yield, and cooking quality of lentils. Field Crop. Res. 1985, 12, 153-161. [CrossRef]

22. Iliadis, C. Influence of genotype and soil type on cooking time in lentil (Lens culinaris Medikus). Short communication. Int. J. Food Sci. Technol. 2003, 38, 89-93. [CrossRef]

23. Vandenberg, A. Postharvest processing and value addition. In The Lentil: Botany, Production and Uses; Erskine, W., Muehlbauer, F., Sarker, A., Sharma, B., Eds.; CABI: Oxford, UK, 2009; pp. 391-408.

24. Hansen, J.R. Hydration of soybean protein: Effect of isolation method and various other parameters on hydration. J. Agric. Food Chem. 1978, 26, 301-304. [CrossRef]

25. Tahir, M.; Lindeboom, N.; Båga, M.; Vandenberg, A.; Ravindra, C.N. Composition and correlation between major seed constituents in selected lentil (Lens culinaris. Medik). Can. J. Plant Sci. 2011, 91, 825-835. [CrossRef]

26. Koutsika-Sotiriou, M.; Mylonas, I.G.; Ninou, E.; Traka-Mavrona, E. The cultivation revival of a landrace: Pedigree and analytical breeding. Euphytica 2010, 176, 15-24. [CrossRef]

27. Stefaniak, T.R.; McPhee, K.E. Lentil. In Grain Legumes, Handbook of Plant Breeding; de Ron, A., Ed.; Springer: New York, NY, USA, 2015; pp. 111-140.

28. Vlachostergios, D.N.; Tzantarmas, C.; Kargiotidou, A.; Ninou, E.; Pankou, C.; Gaintatzi, C.; Mylonas, I.; Papadopoulos, I.; Foti, C.; Chatzivassiliou, E.K.; et al. Single-plant selection within lentil landraces at ultra-low density: A short-time tool to breed high yielding and stable varieties across divergent environments. Euphytica 2018, 214, 58-72. [CrossRef]

29. Fasoula, V.A.; Tokatlidis, I.S. Development of crop cultivars by honeycomb breeding. Agron. Sustain. Dev. 2012, 32, 161-180. [CrossRef] 
30. Tokatlidis, I.S. Crop adaptation to density to optimise grain yield: Breeding implications. Euphytica 2017, 213, 92. [CrossRef]

31. Bishnoi, S.; Khetarpaul, N. Variability in physicochemical properties and nutrient composition of different pea cultivars. Food Chem. 1993, 47, 371-373. [CrossRef]

32. Iliadis, C. Effects of harvesting procedure, storage time and climatic conditions on cooking time of lentils (Lens culinaris Medicus). J. Sci. Food Agric. 2001, 81, 590-593. [CrossRef]

33. AOAC. Official Methods of Analysis, 17th ed.; Association of Official Analytical Chemists: Washington, DC, USA, 2000.

34. Celmeli, T.; Sari, H.; Canci, H.; Sari, D.; Adak, A.; Eker, T.; Toker, C. The Nutritional Content of Common Bean (Phaseolus vulgaris L.) Landraces in Comparison to Modern Varieties. Agronomy 2018, 8, 166. [CrossRef]

35. Iqbal, A.M.; Wani, S.A.; Lone, A.A.; Dar, Z.A.; Nehvi, F.A. Breeding for quality traits in grain legumes. In Conventional and Non-Conventional Interventions in Crop Improvement, 1st ed.; Malik, C.P., Sanghera, G., Wani, S.H., Eds.; M D Publishers: New Delhi, India, 2003.

36. Kargiotidou, A.; Chatzivassiliou, E.K.; Tzantarmas, C.; Tokatlidis, I.S. Seed propagation at low density facilitates the selection of healthy plants to produce seeds with a reduced virus load in a lentil landrace. Seed Sci. Technol. 2015, 43, 31-39. [CrossRef]

37. Taleb, M.H.; Khodambashi, M.; Karimi, M. Study of physical and nutritional quality properties in segregating generations of lentil cross. Int. J. Agric. Crop Sci. 2013, 5, 2740-2742.

38. Solanki, I.S.; Kapoor, A.C.; Singh, U. Nutritional parameters and yield evaluation of newly developed genotypes of lentil (Lens culinaris Medik.). Plant Foods Hum. Nutr. 1999, 54, 79-87. [CrossRef] [PubMed]

39. Boulter, D. The composition and nutritional value of legumes in relationship to crop improvement by breeding. Proc. Nutr. Soc. 1982, 41,1-6. [CrossRef]

40. Chen, Y.; McGee, R.; Vandemark, G.; Brick, M.; Thompson, H.J. Dietary Fiber Analysis of Four Pulses Using AOAC 2011.25: Implications for Human Health. Nutrients 2016, 8, 829. [CrossRef] [PubMed]

41. Kaur, M.; Sandhu, K.S.; Lim, S.T. Microstructure, physicochemical properties and in vitro digestibility of starches from different Indian lentil (Lens culinaris) cultivars. Carbohydr. Polym. 2010, 79, 349-355. [CrossRef]

42. Bhatty, R.S. Composition and Quality of Lentil (Lens culinaris Medik): A Review. Can. Inst. Food Sci. Technol. J. 1988, 21, 144-160. [CrossRef]

43. Dixit, P.; Dubey, D.K. Heritability and genetic advance in induced mutants of lentil (Lens culinaris Med.). Indian J. Genet. Plant Breed. 1985, 45, 520-524.

44. Tambal, H.A.A.; Erskine, W.; Baalbaki, R.; Zaiter, H. Relationship of flower and pod numbers perinflorescence with seed yield in lentil. Exp. Agric. 2000, 36, 369-378. [CrossRef]

45. Bicer, B.T.; Sakar, D. Genetic Variability and Heritability for Grain Yield and Other Characters in Lentil. J. Biol. Sci. 2004, 4, 216-218.

46. Tyagi, S.D.; Khan, M.H. Correlation, path-coefficient and genetic diversity in lentil (Lens culinaris Medik) under rainfed conditions. Int. Res. J. Plant Sci. 2011, 2, 191-200.

47. Hamdi, A.; Erskine, W.; Gates, P. Relationships among economic characters in lentil. Euphytica 1991, 57, 109-116. [CrossRef]

48. Tahir, M.; Vandenberg, A.; Ravindra, C.N. Influence of environment on seed soluble carbohydrates in selected lentil cultivars. J. Food Compost. Anal. 2011, 24, 596-602. [CrossRef]

49. De Almeida Costa, G.E.; da Silva Queiroz-Monici, K.; Pissini Machado Reis, S.M.; de Oliveira, A.C. Chemical composition, dietary fiber and resistant starch contents of raw and cooked pea, common bean, chickpea and lentil legumes. Food Chem. 2006, 94, 327-330. [CrossRef]

50. Tokatlidis, I.; Vlachostergios, D. Sustainable Stewardship of the Landrace Diversity. Diversity 2016, 8, 29. [CrossRef]

(C) 2019 by the authors. Licensee MDPI, Basel, Switzerland. This article is an open access article distributed under the terms and conditions of the Creative Commons Attribution (CC BY) license (http://creativecommons.org/licenses/by/4.0/). 